\title{
Catalytic Ester to Stannane Functional Group Interconversion via Decarbonylative Cross-Coupling of Methyl Esters
}

\author{
Huifeng Yue, Chen Zhu, and Magnus Rueping* \\ Institute of Organic Chemistry, RWTH Aachen University, Landoltweg 1, 52074 Aachen, Germany \\ Supporting Information Placeholder
}

ABSTRACT: An unprecedented conversion of methyl esters to stannanes was realized, providing access to a series of aryl
stannanes via nickel catalysis. Various common esters including ethyl, cyclohexyl, benzyl and phenyl esters can undergo
the newly developed decarbonylative stannylation reaction. The reaction shows broad substrate scope, can differentiate
between different types of esters, and if applied in consecutive fashion allows the transformation of methyl esters into aryl
fluorides or biaryls via flurorination or arylation.

Esters are one of the most ubiquitous classes of organic molecules which exist in a wide range of natural products and synthetic intermediates. ${ }^{1}$ Accordingly, the interconversions between esters and other functional groups are important in organic synthesis. Recently, increasing attention has been devoted to cross coupling reactions using cheap, green and readily available esters as coupling partners instead of commonly used halogenated reagents. ${ }^{2-5}$ In particular, considerable progress has been registered for decarbonylative and decarboxylative reactions of esters to form diverse valuable compounds including organoboron compounds. ${ }^{4,5}$ Although these methods showed great advantages over conventional methodologies, the substrate scope is mainly limited to phenyl esters. Commercially available and inexpensive methyl esters, seemed inefficient in these transformations, which might be due to the higher bond-dissociation energy (Scheme 1). ${ }^{6}$

Scheme 1. Bond-Strength of Acyl C-O Bonds.

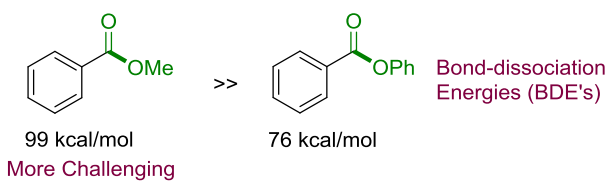

Progress in the activation of methyl esters has been made recently by Garg, Houk and co-workers as well as $\mathrm{Hu}$ and coworkers who described the nickel catalyzed direct amidation of methyl esters with secondary aryl amines and nitroarenes, respectively (Scheme $2 \mathrm{~A}, \mathrm{~B}){ }^{7}$ However, these transformations both underwent a non-decarbonylative process. To the best of our knowledge, the decarbonylative reaction of simple esters such as methyl esters has never been realized. This is surprising since the use of methyl esters would have several advantages including the widespread use and availability of methyl esters as well as the formation and removal of methanol instead of phenols, amides or imides which have so far been obtained as byproducts in decarbonylative processes.

Aryl stannanes are highly important compounds due to their application in the mild and versatile Migita-Kosugi-Stille coupling reaction, ${ }^{8}$ as well as in the synthesis of natural prod- ucts and late-stage functionalization of complex molecules. ${ }^{9}$ Moreover, they are frequently used in the construction of diverse carbon-heteroatom bonds such as $\mathrm{C}-\mathrm{N},{ }^{10} \mathrm{C}-\mathrm{F},{ }^{11}$ and $\mathrm{C}$ $\mathrm{OCF}_{3}{ }^{12}$ bonds. Traditional methods to access stannanes employ the reaction of trialkyltin chloride with air-sensitive organometallic reagents, which may suffer from poor functional group tolerance. ${ }^{13}$ Alternatively, palladium ${ }^{14 \mathrm{a}-\mathrm{c}}$ and nickel ${ }^{14 \mathrm{~d}-\mathrm{g}}$ catalyzed stannylation of different electrophiles as well as $\mathrm{C}-\mathrm{H}$ bond stannylation ${ }^{14 \mathrm{~g}}$ have been developed. One such endeavor developed by Martin group involves nickel-mediated stannylation of phenol derivatives via activation of aryl C-O bonds. ${ }^{14 \mathrm{f}}$

Scheme 2. Nickel catalyzed reactions of methyl esters via non-decarbonylative and decarbonylative pathways.

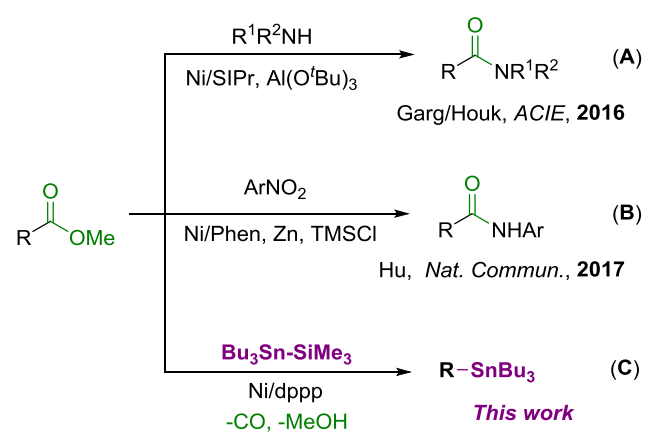

The first ester to stannane interconversion

The first decarbonylative reaction of methyl esters

Given the great importance of aryl stannanes and the limited number of available synthetic methods, it is highly desirable to explore novel and practical methodologies to access these compounds. As part of our continuing efforts in the activation of inert bonds we, herein, report an unprecedented decarbonylative stannylation reaction of simple methyl esters as well as other common esters such as ethyl, cyclohexyl, benzyl and phenyl esters (Scheme 2C). This transformation represents the first decarbonylative reaction of methyl esters 
and features wide substrate scope and broad functional group tolerance.

We started to explore the decarbonylative stannylation of methyl esters by choosing methyl 2-naphthoate (1a) as model substrate in reaction with trimethyl(tributylstannyl)silane 2 . After surveying various ligands, bases, nickel catalysts and solvents, the optimal reaction conditions were assigned as follows: $\mathrm{Ni}(\operatorname{cod})_{2}$, dppp as ligand, $\mathrm{KF}$ as base, $\mathrm{LiCl}$ as additive in toluene at $170^{\circ} \mathrm{C}$ for $48 \mathrm{~h}$ (for details see Table S1).

With the optimized reaction conditions in hand, the scope with respect to the methyl esters was explored (Table 1). A series of methyl esters could be converted into the corresponding products smoothly, regardless of the electronic nature of the substituents on the aromatic ring. Whereas methyl 2-naphthoate (1a) underwent the decarbonylative stannylation reaction in excellent yield, methyl 1-naphthoate (1b) gave the corresponding product in only $52 \%$ yield which was presumably due to steric effects. In contrast to a previous report in which the substrate scope was largely limited to naphthoic acid methyl esters, ${ }^{5 a}$ our newly developed decarbonylative stannylative protocol could be applied to simple benzoic acid methyl esters. For instance, para and even ortho biphenyl carboxylic methyl esters 1c-e could be successfully converted into the corresponding aryl stannanes.

\section{Table 1. Scope of the methyl esters. ${ }^{a, b}$}

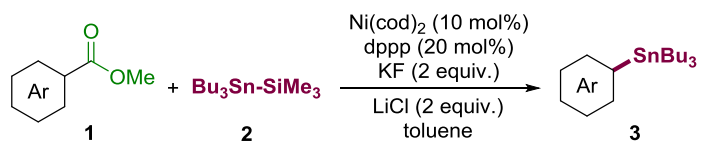

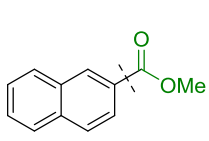

1a, $90 \%$<smiles>COC(=O)c1ccccc1-c1ccccc1</smiles>

$1 d, 50 \%^{c}$<smiles>COC(=O)c1ccc(F)cc1</smiles>

1g, $78 \%$<smiles>COC(=O)c1ccc(C(=O)N(C)c2ccccc2)cc1</smiles>

$1 \mathrm{j}, 70 \%$<smiles>COC(=O)c1cc(OC)cc(OC)c1</smiles>

1m, $80 \%$

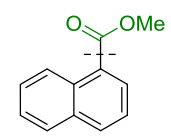

1b $52 \%$<smiles>COC(=O)c1ccc(-c2ccc(C(C)(C)C)cc2)cc1</smiles>

1e, $76 \%^{c}$<smiles>COC(=O)c1cccc(F)c1</smiles>

1h, $96 \%$

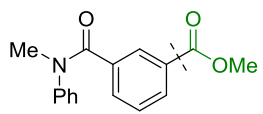

$1 \mathrm{k}, 80 \%$<smiles>COC(=O)c1cncc(/C=C/c2ccccc2)c1</smiles>

1n, $78 \%^{\circ}$<smiles>COC(=O)c1ccc(-c2ccccc2)cc1</smiles>

1c, $53 \%^{c}$<smiles>COC(=O)c1ccc(C)cc1</smiles>

1f, $60 \%$<smiles>COC(=O)c1ccc(C(F)(F)F)cc1</smiles>

1i, $43 \%$

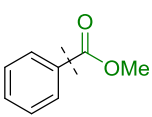

1I, $52 \%$<smiles>COC(=O)c1cccnc1</smiles>

$10,87 \%^{c}$
${ }^{a}$ Reaction conditions: 1 (0.2 mmol), 2 ( $0.3 \mathrm{mmol}, 1.5$ equiv.) $\mathrm{Ni}(\mathrm{cod})_{2}$ (o.02 mmol, $\left.10 \mathrm{~mol} \%\right)$, dppp (0.04 mmol, $20 \mathrm{~mol} \%$ ), $\mathrm{KF}$ (o.4 mmol, 2 equiv.), $\mathrm{LiCl}$ ( $0.4 \mathrm{mmol}, 2$ equiv.) in toluene $(1 \mathrm{ml})$ at $170^{\circ} \mathrm{C}, 48 \mathrm{~h}$. ${ }^{b}$ Yield after purification. ${ }^{c} 72 \mathrm{~h}$.
In addition, the reaction showed a remarkable chemoselectivity, since a wide range of functional groups such as $t$-butyl (1e), methyl (1f), fluoro (1g and 1h), trifluoromethyl (1i), amide (1j and 1k), methoxy (1m), and double bond (1n) were well tolerated. Given the previous reports on the nickel catalyzed $\mathrm{C}-\mathrm{F}, \mathrm{C}-\mathrm{N}$, and $\mathrm{C}-\mathrm{O}$ bond activation, these results show great relevance due to the further functionalization potential of the untouched inert bonds. Moreover, di-substituted substrate 1m could also undergo this protocol in high yield. Notably, pharmaceutically relevant heteroaromatic substrates such as pyridine-derived carboxylic acid methyl esters 1n and 10 were also suitable substrates for this transformation and were converted into the corresponding products in good to excellent yields.

Table 2. Scope of the phenyl esters. ${ }^{a, b}$

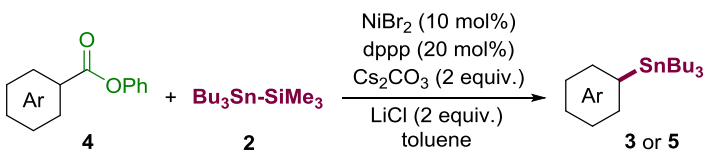

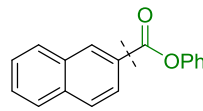

$4 a, 82 \%$<smiles>O=C(Oc1ccccc1)c1ccccc1-c1ccccc1</smiles>

4d, $31 \%$<smiles>O=C(Oc1ccccc1)c1ccc(F)cc1</smiles>

4g, $73 \%$

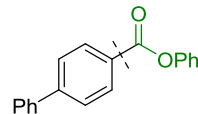

4b, $60 \%$<smiles>CC(C)(C)c1ccc(-c2ccc(C(=O)Oc3ccccc3)cc2)cc1</smiles>

$4 e, 62 \%$

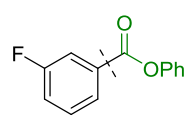

4h, $70 \%$

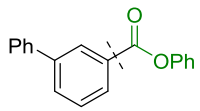

$4 c, 63 \%$<smiles>O=C(c1ccccc1)c1ccc(-c2ccc(F)cc2)cc1</smiles>

$4 f, 54 \%$<smiles>O=C(Oc1ccccc1)c1ccc(C(F)(F)F)cc1</smiles>

$4 i, 60 \%$<smiles>CC(C)(C)c1ccc(C(=O)OC(=O)c2cccc(C(=O)OC(=O)c3cccc(C(=O)OCc4ccccc4)c3)c2)cc1</smiles>

$4 \mathrm{j}, 63 \%$<smiles>CC(C)(C)c1ccc(C(=O)Oc2ccccc2)cc1</smiles><smiles>COc1cc(OC)cc(C(=O)Oc2ccccc2)c1</smiles>

4n, $53 \%$<smiles>COc1ccc(C(=N)/C(=C/C(=O)Oc2ccccc2)C(=O)O)cc1</smiles><smiles>COc1ccc(-c2cncc(C(=O)Oc3ccccc3)c2)cc1</smiles>

$4 p, 50 \%$

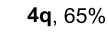<smiles>O=C(Oc1ccccc1)c1ccccc1</smiles>

4o, $70 \%$<smiles>O=C(Oc1ccccc1)c1cccnc1</smiles><smiles>O=C(Oc1ccccc1)c1ccsc1</smiles>

4s, $40 \%$

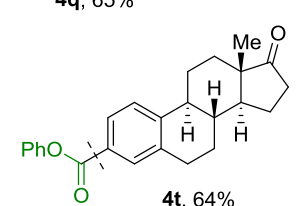

${ }^{a}$ Reaction conditions: 4 ( $\left.0.2 \mathrm{mmol}\right), 2$ (0.3 mmol, 1.5 equiv.), $\mathrm{NiBr}_{2}$ (0.02 mmol, $\left.10 \mathrm{~mol} \%\right)$, dppp (o.04 mmol, $\left.20 \mathrm{~mol} \%\right)$, $\mathrm{Cs}_{2} \mathrm{CO}_{3}$ (0.4 mmol, $20 \mathrm{~mol} \%$ ), LiCl (o.4 mmol, $20 \mathrm{~mol} \%$ ) in toluene $(1 \mathrm{ml})$ at $170^{\circ} \mathrm{C}, 12 \mathrm{~h}$. ${ }^{\circ}$ Yield after purification. 
Furthermore, our catalytic protocol could also be readily extended to aryl carboxylic acid phenyl esters $\mathbf{4}$ by using an inexpensive nickel source $\mathrm{NiBr}_{2}$ as catalyst and $\mathrm{Cs}_{2} \mathrm{CO}_{3}$ as base. Likewise, both naphthoic acid and benzoic acid phenyl esters could undergo the present reaction smoothly, giving the desired products in moderate to high yields. As shown in Table 2, the reaction of phenyl 2-naphthoate (4a) proceeded in $82 \%$ yield. Most of the biphenyl carboxylic acid phenyl esters (4b-f) could be converted into the biphenyl stannanes in good yields. Only substrate $4 \mathbf{d}$ substituted at the ortho position of the aromatic ring led to a lower yield. Again, various functional groups were well tolerated in the new decarbonylative stannylation reaction affording the products in good yields. It is noteworthy that more complex substrates such as estronederived carboxylate $\mathbf{4 t}$ also reacted well.

Table 3. Scope of other simple esters. ${ }^{a, b}$

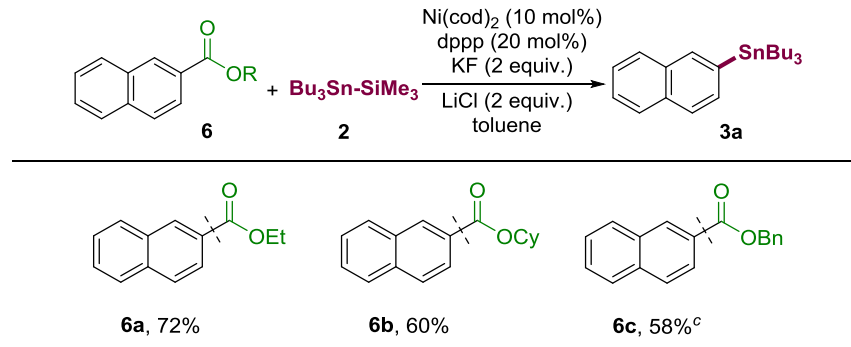

${ }^{a}$ Reaction conditions: 6 (o.2 mmol), 2 (0.3 mmol, 1.5 equiv.), $\mathrm{Ni}(\mathrm{cod})_{2}$ (0.02 mmol, $\left.10 \mathrm{~mol} \%\right)$, dppp (o.04 mmol, $\left.20 \mathrm{~mol} \%\right)$, $\mathrm{KF}$ (0.4 mmol, 2 equiv.), $\mathrm{LiCl}$ (0.4 mmol, 2 equiv.) in toluene $(1 \mathrm{ml})$ at $170{ }^{\circ} \mathrm{C}, 48 \mathrm{~h}$. ${ }^{b}$ Yield after purification. ${ }^{c}$ Reaction conditions as for phenyl esters were used, $24 \mathrm{~h}$.

Scheme 3. a) Selective stannylation of phenyl ester with methyl ester untouched. b) Monostannylation/fluorination and arylation of bismethyl esters.

a) Selective stannylation of phenyl ester with methyl ester untouched

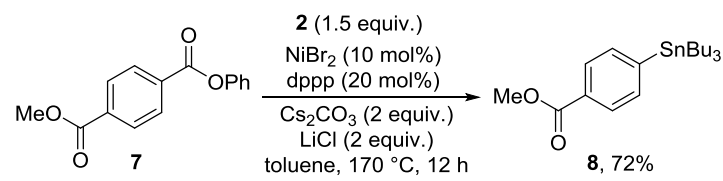

b) Mono-stannylation/fluorination and arylation of bis-methyl esters

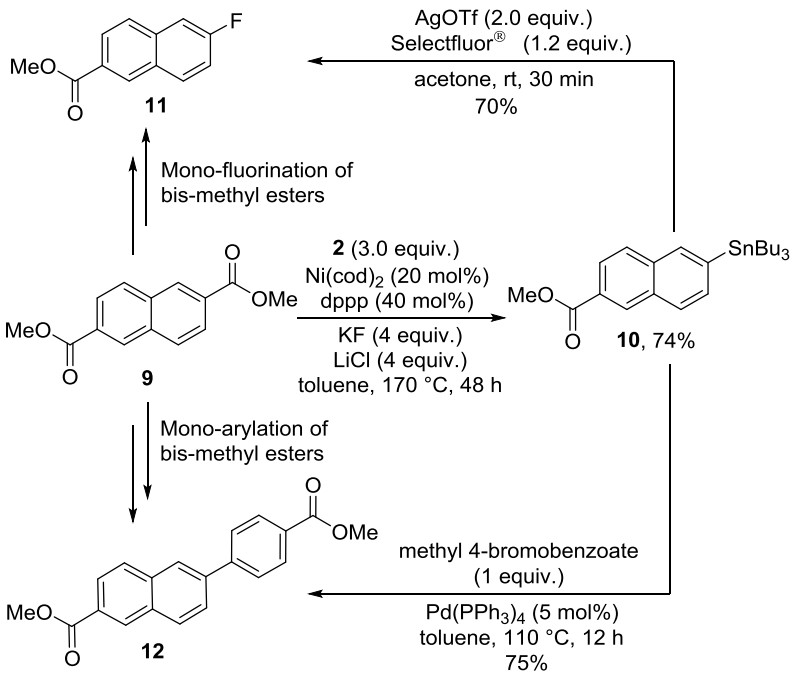

In addition to methyl and phenyl esters, other types of simple esters were also evaluated. Ethyl, cyclohexyl and benzyl ester derivatives 6a-c could all be converted into 3a in good yields, indicating the advantages of our newly developed decarbonylative stannylation protocol (Table 3). To explore whether this new methodology could efficiently differentiate between two different types of esters, we subjected substrate $\mathbf{7}$ bearing both methyl and phenyl ester groups to the present reaction conditions. Gratifyingly, substrate 7 underwent this transformation with excellent chemoselectivity and the phenyl ester group was selectively stannylized, delivering product $\mathbf{8}$ bearing the methyl ester untouched, in good yield (Scheme 3a). Interestingly, mono-stannylation of substrate 9 bearing two methyl ester groups was also realized, affording product 10 in good yield along with trace amount of the double stannylated product. More importantly, further reaction of 10 with Selectfluor or methyl 4-bromobenzoate gave the mono-fluorination or mono-arylation product (11 and 12) of bis-methyl ester 9, thus enabling the challenging interconversion between methyl esters and other important functional groups (Scheme $3 \mathrm{~b}$ ).

In summary, the functional group interconversion of inert methyl esters into stannanes was realized for the first time with the aid of nickel catalysis. A series of methyl esters as well as other common esters such as ethyl, cyclohexyl, benzyl and phenyl esters all underwent this decarbonylative stannylation protocol smoothly, affording diverse aryl stannanes which are important in the construction of C-C and C-heteroatom bonds. Moreover, this method could efficiently differentiate between two different types of esters. In addition, the mono-stannylation/fluorination and monostannylation/arylation of bis-methyl esters could be realized, showing its great practical value. Furthermore, the stannylation protocol shows good chemoselectivity and functional groups including groups previously used in cross-couplings remain intact. Given that aryl stannanes are highly valuable products for different applications this new ester to stannane interconversion will be of value and may become a good alternative to aryl halide stannylation reactions. Efforts to investigate the mechanism and to broaden the scope further are currently ongoing in our laboratories and will be reported in due course.

\section{ASSOCIATED CONTENT}

Supporting Information. Detailed experimental procedures, spectral data for all compounds, and copies of ${ }^{1} \mathrm{H},{ }^{13} \mathrm{C},{ }^{19} \mathrm{~F}$, and ${ }^{119}$ Sn NMR spectra. This material is available free of charge via the Internet at http://pubs.acs.org.

\section{AUTHOR INFORMATION}

\section{Corresponding Author \\ * magnus.rueping@rwth-aachen.de}

\section{Author Contributions}

The manuscript was written through contributions of all authors. / All authors have given approval to the final version of the manuscript.

\section{ACKNOWLEDGMENT}


H.Y. was supported by the China Scholarship Council.

\section{REFERENCES}

(1) (a) Trost, B. M.; Fleming, I. Comprehensive Organic Synthesis, Vol. 6, Pergamon Press, New York, 1992. (b) Larock, R. C. Comprehensive Organic Transformations, 2 ed., Wiley-VCH, New York, 1999. (c) Smith, M. B. in Compendium of Organic Synthetic Methods, Vol. 9, Wiley, New York, 2001, p. 100.

(2) For selected recent examples of aryl $\mathrm{C}-\mathrm{O}$ bond activation reactions of esters, see: (a) Quasdorf, K. W.; Tian, X.; Garg, N. K. J. Am. Chem. Soc. 20o8, 130, 14422-14423. (b) Li, B. J.; Li, Y. Z.; Lu, X. Y.; Liu, J.; Guan, B. T.; Shi, Z. J. Angew. Chem. Int. Ed. 2oo8, 47, 10124-10127. (c) Guan, B.-T.; Wang, Y.; Li, B.-J.; Yu, D.-G.; Shi, Z.-J. J. Am. Chem. Soc. 20o8, 130, 14468-14470. (d) Li, B.-J.; Xu, L.; Wu, Z.-H.; Guan, B.-T.; Sun, C.-L.; Wang, B.-Q.; Shi, Z.-J. J. Am. Chem. Soc. 2009, 131, 14656-14657. (e) Molander, G. A.; Beaumard, F. Org. Lett. 2010, 12, 4022-4025. (f) Shimasaki, T.; Tobisu, M.; Chatani, N. Angew. Chem. Int. Ed. 2010, 49, 29292932. (g) Muto, K.; Yamaguchi, J.; Itami, K. J. Am. Chem. Soc. 2011, 134, 169-172. (h) Ehle, A. R.; Zhou, Q.; Watson, M. P. Org. Lett. 2012, 14, 1202-1205. (i) Yang, J.; Chen, T.; Han, L.-B. J. Am. Chem. Soc. 2015, 137, 1782-1785. (j) Takise, R.; Muto, K.; Yamaguchi, J.; Itami, K. Angew. Chem. Int. Ed. 2014, 53, 6791-6794. (k) Kinuta, H.; Hasegawa, J.; Tobisu, M.; Chatani, N. Chem. Lett. 2014, 44, 366-368. (1) Zarate, C.; Martin, R. J. Am. Chem. Soc. 2014, 136, 2236-2239. (m) Correa, A.; León, T.; Martin, R. J. Am. Chem. Soc. 2014, 136, 1062-1069. (n) Correa, A.; Martin, R. J. Am. Chem. Soc. 2014, 136, 7253-7256. (o) Cornella, J.; Jackson, E. P.; Martin, R. Angew. Chem. Int. Ed. 2015, 54, 4075-4078. (p) Takise, R.; Itami, K.; Yamaguchi, J. Org. Lett. 2016, 18, 4428-4431. (q) Guo, L.; Hsiao, C.-C.; Yue, H.; Liu, X.; Rueping, M. ACS Catal. 2016, 6, 4438-4442. (r) Yue, H.; Guo, L.; Liu, X.; Rueping, M. Org. Lett. 2017, 19, 1788-1791. For theoretical studies, see: (s) Li, Z.; Zhang, S.-L.; Fu, Y.; Guo, Q.-X.; Liu, L. J. Am. Chem. Soc. 20o9, 131, 8815-8823. (t) Muto, K.; Yamaguchi, J.; Lei, A.; Itami, K. J. Am. Chem. Soc. 2013, 135, 16384-16387. (u) Xu, H.; Muto, K.; Yamaguchi, J.; Zhao, C.; Itami, K.; Musaev, D. G. J. Am. Chem. Soc. 2o14, 136, 14834-14844.

(3) Examples of acyl $\mathrm{C}-\mathrm{O}$ bond activations of esters via nondecarbonylative pathway, see: (a) Kakino, R.; Shimizu, I.; Yamamoto, A. Bull. Chem. Soc. Jpn. 2oo1, 74, 371-376. (b) Tatamidani, H.; Kakiuchi, F.; Chatani, N. Org. Lett. 2004, 6, 3597-3599. (c) Tatamidani, H.; Yokota, K.; Kakiuchi, F.; Chatani, N. J. Org. Chem. 2004, 69, 5615-5621. (d) Ben Halima, T.; Vandavasi, J. K.; Shkoor, M.; Newman, S. G. ACS Catal. 2017, 7, 2176-218o. (e) Halima, T. B.; Zhang, W.; Yalaoui, I.; Hong, X.; Yang, Y.-F.; Houk, K. N.; Newman, S. G. J. Am. Chem. Soc. 2017, 139, 1311-1318.

(4) For examples of acyl $\mathrm{C}-\mathrm{O}$ bond activation reactions of esters via decarbonylative pathway, see: (a) Gooßen, L. J.; Paetzold, J. Angew. Chem. Int. Ed. 2002, 41, 1237-1241. (b) Gribkov, D. V.; Pastine, S. J.; Schnürch, M.; Sames, D. J. Am. Chem. Soc 2007, 129, 11750-11755. (c) Amaike, K.; Muto, K.; Yamaguchi, J.; Itami, K. J. Am. Chem. Soc. 2012, 134, 13573-13576. (d) Meng, L.; Kamada, Y.; Muto, K.; Yamaguchi, J.; Itami, K. Angew. Chem. Int. Ed. 2013, 52, 10048-10051. (e) Muto, K.; Yamaguchi, J.; Musaev, D. G.; Itami, K. Nat. Commun. 2015, 6, 7508. (f) LaBerge, N. A.; Love, J. A. Eur. J. Org. Chem. 2015, 2015, 5546-5553. (g) Desnoyer, A. N.; Friese, F. W.; Chiu, W.; Drover, M. W.; Patrick, B. O.; Love, J. A. Chem. Eur. J. 2016, 22, 4070-4077. (h) Guo, L.; Chatupheeraphat, A.; Rueping, M. Angew. Chem. Int. Ed. 2016, 55, 11810-11813. (i) Guo, L.; Rueping, M. Chem. Eur. J. 2016, 22, 16787-1679o. (j) Pu, X.; Hu, J.; Zhao, Y.; Shi, Z. ACS Catal. 2016, 6, 6692-6698. (k) Amaike, K.; Itami, K.; Yamaguchi, J. Chem. Eur. J. 2016, 22, 43844388. (l) Takise, R.; Isshiki, R.; Muto, K.; Itami, K.; Yamaguchi, J. J. Am. Chem. Soc. 2017, 139, 3340-3343. (m) Yue, H.; Guo, L.; Liao, H. H.; Cai, Y.; Zhu, C.; Rueping, M. Angew. Chem. Int. Ed. 2017,
56, 4282-4285. (n) Yue, H.; Guo, L.; Lee, S. C.; Liu, X.; Rueping, M. Angew. Chem. Int. Ed. 2017, 56, 3972-3976. (o) Liu, X.; Jia, J.; Rueping, M. ACS Catal. 2017, 7, 4491-4496. (p) Chatupheeraphat, A.; Liao, H.-H.; Lee, S.-C.; Rueping, M. Org. Lett. 2017, 19, 42554258. (q) Okita, T.; Kumazawa, K.; Takise, R.; Muto, K.; Itami, K.; Yamaguchi, J. Chem. Lett. 2017, 46, 218-22o. For theoretical studies, see: (r) Hong, X.; Liang, Y.; Houk, K. J. Am. Chem. Soc. 2014, 136, 2017-2025. (s) Lu, Q.; Yu, H.; Fu, Y. J. Am. Chem. Soc. 2014, 136, 8252-8260. For a recent review, see: (t) Takise, R.; Muto, K.; Yamaguchi, J. Chem. Soc. Rev. 2017, 46, 5864-5888.

(5) (a) Fawcett, A.; Pradeilles, J.; Wang, Y.; Mutsuga, T.; Myers, E. L.; Aggarwal, V. K. Science 2017, 357, 283-286. (b) Li, C.; Wang, J.; Barton, L. M.; Yu, S.; Tian, M.; Peters, D. S.; Kumar, M.; Yu, A. W.; Johnson, K. A.; Chatterjee, A. K.; Yan, M.; Baran, P. S. Science 2017, 356, eaam7355. (c) Hu, D.; Wang, L.; Li, P., Org. Lett. 2017, 19, 2770-2773.

(6) Luo, Y.-R. Comprehensive Handbook of Chemical Bond Energies, CRC Press: Boca Raton, FL, 2007.

(7) (a) Hie, L.; Fine Nathel, N. F.; Hong, X.; Yang, Y. F.; Houk, K. N.; Garg, N. K. Angew. Chem. Int. Ed. 2016, 55, 2810-2814. (b) Cheung, C. W.; Ploeger, M. L.; Hu, X. Nat. Commun. 2017, 8, 14878.

(8) (a) Stille, J. K. Angew. Chem. Int. Ed. 1986, 25, 508-524. (b) Hassan, J.; Sevignon, M.; Gozzi, C.; Schulz, E.; Lemaire, M. Chem. Rev. 2002, 102, 1359-1470. (c) Espinet, P.; Echavarren, A. M. Angew. Chem. Int. Ed. 2004, 43, 4704-4734. (d) Johansson Seechurn, C. C.; Kitching, M. O.; Colacot, T. J.; Snieckus, V. Angew. Chem. Int. Ed. 2012, 51, 5062-5085.

(9) (a) Ragan, J.; Raggon, J.; Hill, P.; Jones, B.; McDermott, R.; Munchhof, M.; Marx, M.; Casavant, J.; Cooper, B.; Doty, J. Org. Process Res. Dev. 2003, 7, 676-683. (b) Alonso, E.; Fuwa, H.; Vale, C.; Suga, Y.; Goto, T.; Konno, Y.; Sasaki, M.; LaFerla, F. M.; Vieytes, M. R.; Giménez-Llort, L. J. Am. Chem. Soc. 2012, 134, 7467-7479. (c) Valot, G.; Regens, C. S.; O'Malley, D. P.; Godineau, E.; Takikawa, H.; Fürstner, A. Angew. Chem. Int. Ed. 2013, 52, 9534-9538. (d) Li, J.; Yang, P.; Yao, M.; Deng, J.; Li, A. J. Am. Chem. Soc. 2014, 136, 16477-1648o. (e) Mailhol, D.; Willwacher, J.; Kausch-Busies, N.; Rubitski, E. E.; Sobol, Z.; Schuler, M.; Lam, M.-H.; Musto, S.; Loganzo, F.; Maderna, A. J. Am. Chem. Soc. 2014, 136, 15719-15729. (f) Logan, M. M.; Toma, T.; Thomas-Tran, R.; Du Bois, J. Science 2016, 354, 865-869.

(10) Lam, P. Y.; Vincent, G.; Bonne, D.; Clark, C. G. Tetrahedron Lett. 2002, 43, 3091-3094.

(11) (a) Furuya, T.; Strom, A. E.; Ritter, T. J. Am. Chem. Soc. 2009, 131, 1662-1663. (b) Tang, P.; Furuya, T.; Ritter, T. J. Am. Chem. Soc. 2010, 132, 12150-12154. (c) Ye, Y.; Sanford, M. S. J. Am. Chem. Soc. 2013, 135, 4648-4651. (d) Gamache, R. F.; Waldmann, C.; Murphy, J. M. Org. Lett. 2016, 18, 4522-4525.

(12) Huang, C.; Liang, T.; Harada, S.; Lee, E.; Ritter, T. J. Am. Chem. Soc. 2011, 133, 13308-13310.

(13) (a) Soderquist, J. A.; Hassner, A. J. Am. Chem. Soc. 1980, 102, 1577-1583. (b) Knochel, P.; Singer, R. D. Chem. Rev. 1993, 93, 2117-2188. (c) Gosmini, C.; Périchon, J. Org. Biomol. Chem. 2005, 3, 216-217.

(14) (a) Azizian, H.; Eaborn, C.; Pidcock, A. J. Organomet. Chem. 1981, 215, 49-58. (b) Corcoran, E. B.; Williams, A. B.; Hanson, R. N. Org. Lett. 2012, 14, 4630-4633. (c) Wulff, W. D.; Peterson, G. A.; Bauta, W. E.; Chan, K.-S.; Faron, K. L.; Gilbertson, S. R.; Kaesler, R. W.; Yang, D. C.; Murray, C. K. J. Org. Chem. 1986, 51, 277-279. (d) Komeyama, K.; Asakura, R.; Takaki, K. Org. Biomol. Chem. 2015, 13, 8713-8716. (e) Shirakawa, E.; Nakao, Y.; Yoshida, H.; Hiyama, T. J. Am. Chem. Soc. 2000, 122, 9030-9031 (f) Gu, Y.; Martín, R. Angew. Chem. Int. Ed. 2017, 56, 3187-3190. (g) Doster, M. E.; Hatnean, J. A.; Jeftic, T.; Modi, S.; Johnson, S. A. J. Am. Chem. Soc. 2010, 132, 11923-11925. (h) Qiu, D.; Meng, H.; Jin, L.; Wang, S.; Tang, S.; Wang, X.; Mo, F.; Zhang, Y.; Wang, J. Angew. Chem. Int. Ed. 2013, 52, 11581-11584. 


\section{Insert Table of Contents artwork here}

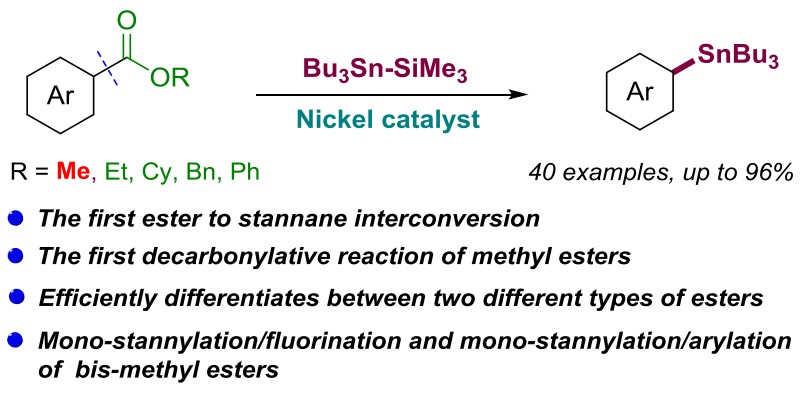

